\title{
A Rare Case of Trocar Site Endometriosis managed Laparoscopically
}

\author{
${ }^{1}$ Anushree Mittal, ${ }^{2}$ Rahul Manchanda, ${ }^{3} \mathrm{BC}$ Manjula, ${ }^{4}$ Soma Ghoshal
}

\begin{abstract}
Abdominal wall endometriosis, also known as scar endometriosis, is extremely rare and mainly occurs at surgical scar sites. To our knowledge, 15 case reports related to trocar site endometriosis have been published in the English language and literature to date. The traditional age old management of scar endometriosis has been wide local excision leaving a healthy margin, but recently laparoscopy has emerged out to be the management of choice even in cases of scar endometriosis. Herein, we present the case of a 39-year-old woman (who had been previously operated on for left ovarian endometrioma 11 years ago by laparoscopy) with the complaint of a painful mass at the right suprapubic trocar site in a cyclic pattern. Consequently, the scar endometriosis was excised laparoscopically, following which the patient was completely relieved of her symptoms.
\end{abstract}

Keywords: Trocar site endometriosis, Laparoscopic excision, Abdominal wall endometriosis.

How to cite this article: Mittal A, Manchanda R, Manjula BC, Ghoshal S. A Rare Case of Trocar Site Endometriosis managed Laparoscopically. J South Asian Feder Menopause Soc 2014;2(2):91-94.

\section{Source of support: Nil}

Conflict of interest: None

\section{INTRODUCTION}

Endometriosis is defined as the presence of functioning endometrial glands and stroma outside the usual location in the lining of the uterine cavity. ${ }^{1}$ Endometriomas have been found in association with surgical scars from a variety of procedures. With the increased use of laparoscopy, a few case reports have described abdominal wall endometriomas at port sites. ${ }^{2-4}$ This study presents the case of a patient with secondary trocar site endometriosis, who previously had undergone laparoscopic resection of a left ovarian endometriotic cyst. The rarity involved in this case is based upon two aspects, one

\footnotetext{
1,3,4 Fellow, ${ }^{2}$ Consultant

${ }^{1-4}$ Department of Gynecology and Endoscopy, Pushpawati Singhania Research Institute, New Delhi, India
}

Corresponding Author: Anushree Mittal, Fellow, Department of Gynecology and Endoscopy, Pushpawati Singhania Research Institute, New Delhi, India, Phone: 09582632341 , e-mail: anushree. mittal02@gmail.com being the development of trocar site endometriosis after the removal of a previous ovarian endometrioma and the second being complete surgical management of scar endometriosis by laparoscopic approach, which in itself is rare and has not been mentioned in the literature as per our knowledge. Blanco et $\mathrm{al}^{5}$ described 12 cases of abdominal wall endometriomas with only one having a preexisting endometriosis, while in another series of 17 patients, associated pelvic endometriosis was present in as much as $24 \%$ of the cases. ${ }^{6}$

\section{CASE REPORT}

A 39-year-old female P1L1A1, married for 14 years, presented to our outpatient clinic with complaints of right lower quadrant abdominal pain for 2 months. Pain was acute, excruciating and had a cyclical pattern. Her medical history included a laparoscopic endometrioma resection 11 years back, one cesarean section 7 years back and curettage for missed abortion 6 years back. Her complains of severe dysmenorrhea associated with tenesmus dates back 18 years back, for which she was prescribed oral contraceptive pills for 3 to 4 years, but was not relieved. Menstrual cycles were regular. In 2003 (11 years back), she underwent laparoscopic resection of a $4 \times 5 \mathrm{~cm}$ left sided endometrioma with fulguration of few endometriotic implants in the pouch of Douglas. Histopathological evaluation of the cystectomy material had confirmed the diagnosis of endometriosis, and the patient was placed on medical therapy. She was on oral contraceptive pills for another 4 years but symptoms persisted. On this admission, she stated that pain in the abdominal wall commenced a few months after the laparoscopic surgery, was cyclical in nature and had worsened progressively over the previous 2 months, to an extent that pain was relieved only on injectable analgesics. Pain was localized in the right lower quadrant near the previous trocar site and would exaggerate on 3rd to 4 th days of the cycle.

On inspection, two healed scars compatible with trocar port sites: one inferior to the umbilicus (per umbilical trocar, $10 \mathrm{~mm}$ ) and the other on the right lower quadrant (right side trocar, $5 \mathrm{~mm}$ ) was observed. On palpation, localized tenderness over the right lateral suprapubic trocar site was felt. Routine biochemical tests and CA125 were within normal ranges. Abdominal computed 
tomography scan showed multiple subcentimeter sized contiguous peripherally enhancing nodules in the right iliac fossa region, lateral to cecum with thickened parietal peritoneum. On corroborative T1W MR axial sections, multiple tiny hyperintense nodules consistent with endometriotic deposits in the right iliac fossa, was found.

Patient was planned for laparoscopic exploration under general anesthesia. Findings on laparoscopy showed a large port site endometriotic mass in the right anterior abdominal wall $(5 \times 6 \mathrm{~cm})$, adjacent to the round ligament, fixed to the surrounding tissues which was extending into the posterior rectus fascia (Fig. 1). There were few endometriotic implants in the pouch of Douglas with dense adhesions. Bilateral tubes and ovaries were stuck in ovarian fossa. Adhesiolysis was done and anatomy restored. Endometriotic areas were cauterized. Extensive dissection of the mass was done (Fig. 2). The underlying fascia and the muscle that contained palpable tumefactions were also excised (Fig. 3), and port site closed. Pathologic examination revealed foci

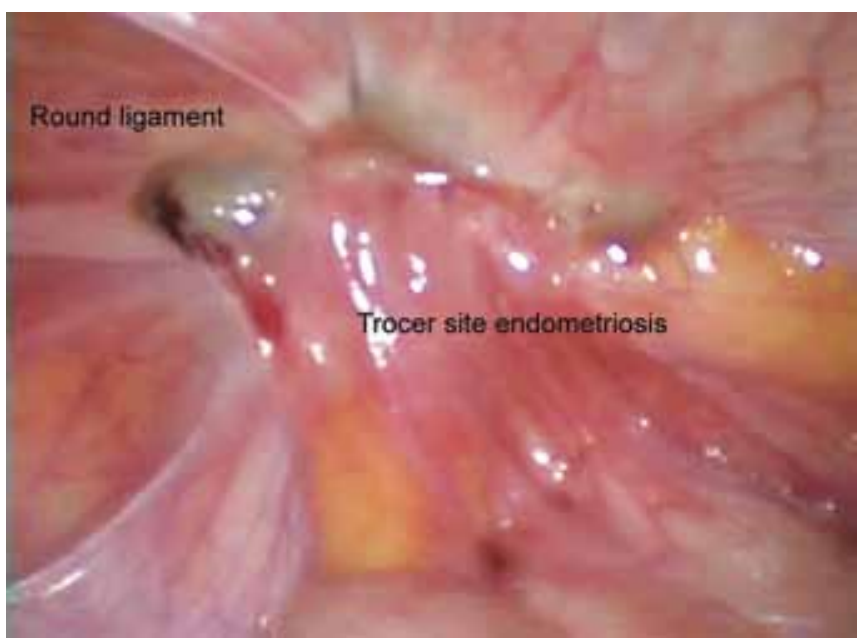

Fig. 1: Initial view of trocar scar endometriosis showing its position in relation to round ligament

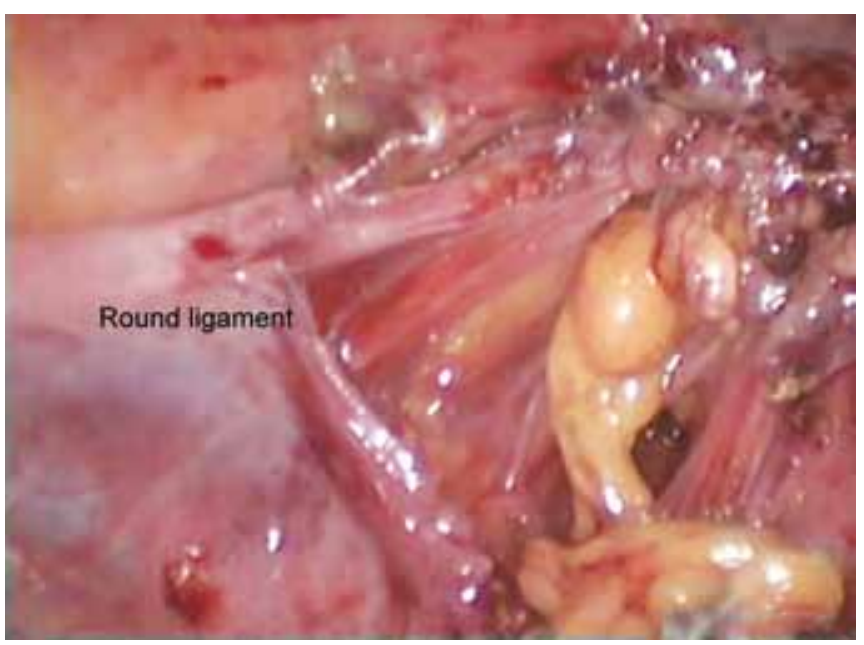

Fig. 2: Partially dissected lesion of endometriosis comprising of endometrial glands and stroma within connective tissue, along with hemosiderinladen macrophages. The patient was put on GnRH agonist once a month for 6 months. On further follow up, she was completely relieved of her symptoms and resumed her daily activities from 3rd postoperative day.

\section{DISCUSSION}

Endometriosis, first described by Rokitansky in 1861, is a common benign gynecologic disorder defined as ectopic implantation of endometrial glands and stroma outside the uterine cavity. ${ }^{3}$ Endometriosis can also be classified as pelvic or extrapelvic according to its location. Pelvic endometriosis includes lesions of the fallopian tubes, ovaries, and pelvic peritoneum. Extrapelvic endometriosis refers to endometriotic implants found in other areas of the body, including the gastrointestinal tract, pulmonary structures, urinary system, abdominal wall, skin, and even the central nervous system. With the introduction of laparoscopy into both general and gynecologic surgery over the past 20 years, many complications associated with trocar port sites have been reported. Though the most frequent complication is an incisional hernia, especially with large-diameter trocars, tumor cell seeding along the trocar tract and trocar site endometriosis are two rare complications. ${ }^{7}$ In searches of the PubMed, Google Scholar, and Medline databases using the search terms 'endometriosis,' 'scar endometriosis,' 'abdominal wall endometriosis,' and 'trocar' alone and, in various combinations, very few English-language articles on trocar site endometriosis were found. Denton et $\mathrm{al}^{8}$ reported the first case of trocar site endometriosis in 1990. The first reported case of abdominal wall endometrioma in laparoscopic trocar tract after a previous laparoscopic resection of endometrioma was by Healy et al. ${ }^{9}$

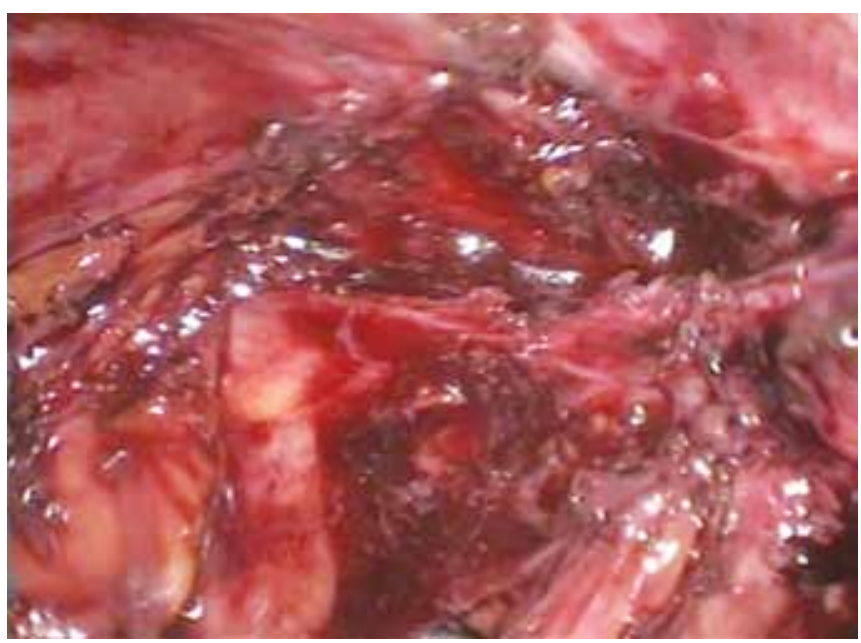

Fig. 3: Abdominal wall dissected intraperitoneally lying bereft of endometriotic tissue 
The precise etiopathogenesis of endometriosis remains controversial, and many theories have been proposed, including cellular immunity, coelomic metaplasia, implantation or retrograde menstruation, vascular and lymphatic metastasis, dissemination, and direct transplantation.,10 The etiopathogenetic mechanism by which endometriosis develops at a surgical wound site is evidently linked to endometrial tissue dissemination during the course of gynecological surgery. It has also been suggested that localized tissue ischemia renders that tissue conducive to implantation. ${ }^{3,11}$ Also, the practice of insufflating $\mathrm{CO}_{2}$ into the peritoneal cavity causes cell aerosolization and may promote tumor cell shedding (aerosolization theory). ${ }^{3}$

Diagnosis of endometriosis arising from an abdominal wall scar, such as a trocar site, can be detected using computed tomography (CT), ultrasonography (USG), ultrasound (US)-guided fine-needle aspiration (FNA) and magnetic resonance imaging (MRI). ${ }^{12,13}$ On USG, the masses appear as solid, hypoechoic lesions in the abdominal wall that contain internal vascularity on power Doppler examination..$^{12}$ The CT and MRI characteristics of abdominal wall endometriosis are nonspecific, with both showing a solid enhancing mass in the abdominal wall. Although useful, CT, USG, and MRI cannot provide a definitive preoperative diagnosis. In our case, MRI showed a peritoneal thickening which in some circumstances can actually be due to inflammatory changes, hemangiomas, foreign body reaction, mesothelial hyperplasia, and hemosiderin deposits rather than endometriosis. ${ }^{13}$ However, laparoscopy remains the mainstay for both diagnosis as well as management of endometriosis. Though final conclusion is based on histopathology.

The differential diagnosis of surgical scar endometriosis is broad and often confused with other pathologic conditions, such as a suture granuloma, abscess, inguinal or incisional hernia, soft-tissue sarcoma, desmoid tumor, lipoma, metastatic tumor and sebaceous cyst. Therefore, the pathologic diagnosis of endometriosis should be confirmed. ${ }^{2}$

Historically, two major approaches exist for management of endometriosis: medical and surgical. Medical treatments are focused on hormonal manipulation of the menstrual cycle. Although hormonal modulation may be useful in some settings like preoperative preparation, it rarely yields satisfactory long-term results. This may be because of fibrotic changes and scar tissue, resulting in pelvic pain that is refractory to medical therapy. ${ }^{14}$ Currently, laparotomic wide local excision of endometriotic lesions appears to be the most effective treatment. But, a prospective, randomized controlled trial evaluating laparotomy versus laparoscopy for the treatment of endometriomas revealed that laparoscopy is associated with lower analgesic requirement, earlier discharge and shorter postoperative recovery time. Laparoscopy also offers improved visualization of the abdomen and pelvis, with the ability to magnify and approach structures from angles not accessible by laparotomy, allowing for a more thorough treatment of extensive disease, such as scar endometriosis. ${ }^{15}$ Safe laparoscopic excision of an endometriotic lesion requires acute awareness of surrounding structures.

\section{CONCLUSION}

Abdominal wall endometrioma is more common than generally assumed in patients visiting the hospital. In patients with chronic pelvic pain near surgical scars associated with cyclic pattern, a thorough history and physical examination are sufficient to establish the presence of endometrioma. Laparoscopic wide local excision is the treatment modality. Laparoscopy affords easy, effective and definitive method for diagnosis and complete resection of endometriosis even in case of scar endometriosis and also defines the borders of dissection very clearly due to enhanced vision.

\section{ACKNOWLEDGMENT}

I am using this opportunity to express my gratitude to everyone who supported me throughout the course of making this paper/case report. I would like to express the deepest appreciation to my mentor Dr Rahul Manchanda who has shown the attitude and the substance of a genius: he continually and persuasively conveyed a spirit of adventure in regard to clinical skills as well as academics, and an excitement in regard to teaching. Without his supervision and constant help this dissertation would not have been possible. His willingness to motivate us contributed tremendously to my project. I am sincerely grateful to him for sharing his truthful and illuminating views on a number of issues related to the topic.

I take this opportunity to express my gratitude to my cofellows Dr Manjula BCs and Dr Soma Ghoshal, who have been instrumental in the successful completion of this paper. They willingly helped me gather the necessary data and information needed for this compilation.

I would like to thank my loved ones-my parents and my in-laws, who have supported me throughout the entire process. A special mention to my husband Dr Amit Pande, both for keeping me harmonious and helping me putting pieces together. I will be grateful forever for your love.

\section{REFERENCES}

1. Akbulut S, Dursun P, Kocbiyik A, Harman A, Sevmis S. Appendiceal endometriosis presenting as perforated appendicitis: report of a case and review of literature. Arch Gynecol Obstet 2009;280(3):495-497. 
2. Emre A, Akbulut S, Yilmaz M, Bozdag Z. Laparoscopic trocar port site endometriosis: a case report and brief literature review. Int Surg 2012;97(2):135-139.

3. Martínez-Serna T, Stalter KD, Filipi CJ, Tomonaga T. An unusual case of endometrial trocar site implantation. Surg Endosc 1998;12:992-994.

4. Strelec M, Dmitrovic R, Matkovic S. Trocar scar endometriosis. Gynaecol Perinatol 2009;188(1):34-35.

5. Blanco RG, ParithivelVS,Shah AK, GumbsMA,Schein M, GerstPH. Abdominal wall endometriomas. Am J Surg 2003; 185(6):596-598.

6. Chatterjee SK. Scar endometriosis: a clinicopathologic study of 17 cases. Obstet Gynecol 1980;56(1):81-84.

7. Sirito R, Puppo A, Centurioni MG, Gustavino C. Incisional hernia on the 5-mm trocar port site and subsequent wall endometriosis on the same site: a case report. Am J Obstet Gynecol 2005;193(3, pt 1):878-880.

8. Denton GW, Schofield JB, Gallagher P. Uncommon complications of laparoscopic sterilization. Ann R Coll Surg Engl 1990;72(3):210-211.

9. Healy JT, Wilkinson NW, Sawyer M. Abdominal wall endometrioma in a laparoscopic trocar tract: a case report.Am Surg 1995;61(11):962-963.
10. Hensen JH, Van Breda Vriesman AC, Puylaert JB. Abdominal wall endometriosis: clinical presentation and imaging features with emphasis on sonography. Am J Roentgenol 2006;186(3):616-620.

11. Lee HH, Lim S, Shin JW, Park CY. A case of trocar site implantation of endometriosis three years after laparoscopic hysterectomy. Korean J Obstet Gynecol 2012;55(4):290-292.

12. Savelli L, Manuzzi L, Di Donato N, Salfi N, Trivella G, Ceccaroni M, Seracchioli R. Endometriosis of the abdominal wall: ultrasonography and Doppler characteristics. Ultrasound Obstet Gynecol 2012;39(3):336-340.

13. Hsu AL, Khachikyan I, Stratton P. Invasive and non-invasive methods for the diagnosis of endometriosis. Clin Obstet Gynecol 2010 Jun;53(2):413-419.

14. Nezhat C, Hajhosseini B, King LP. Laparoscopic management of bowel endometriosis: predictors of severe disease and recurrence. JSLS 2011 Oct-Dec;15(4):431-438.

15. Schipper E, Nezhat C. Video-assisted laparoscopy for the detection and diagnosis of endometriosis. Int $\mathrm{J}$ Women's Health 2012;4:383-393. Published online Jul 31, 2012. 